\title{
The dynamic cardiac biosimulator: A method for training physicians in beating-heart mitral valve repair procedures
}

\author{
Alberto M. Leopaldi, PhD, ${ }^{\text {a }}$ Krzysztof Wrobel, MD, ${ }^{b}$ Giovanni Speziali, MD, Sjoerd van Tuijl, BSc, ${ }^{a}$ \\ Agne Drasutiene, $\mathrm{MD},{ }^{\mathrm{c}}$ and W. Randolph Chitwood, Jr, $\mathrm{MD}^{\mathrm{d}}$
}

\section{ABSTRACT}

Objective: Previously, cardiac surgeons and cardiologists learned to operate new clinical devices for the first time in the operating room or catheterization laboratory. We describe a biosimulator that recapitulates normal heart valve physiology with associated real-time hemodynamic performance.

Methods: To highlight the advantages of this simulation platform, transventricular extruded polytetrafluoroethylene artificial chordae were attached to repair flail or prolapsing mitral valve leaflets. Guidance for key repair steps was by 2-dimensional/3-dimensional echocardiography and simultaneous intracardiac videoscopy.

Results: Multiple surgeons have assessed the use of this biosimulator during artificial chordae implantations. This simulation platform recapitulates normal and pathologic mitral valve function with associated hemodynamic changes. Clinical situations were replicated in the simulator and echocardiography was used for navigation, followed by videoscopic confirmation.

Conclusions: This beating heart biosimulator reproduces prolapsing mitral leaflet pathology. It may be the ideal platform for surgeon and cardiologist training on many transcatheter and beating heart procedures. ( $\mathrm{J}$ Thorac Cardiovasc Surg 2018;155:147-55)

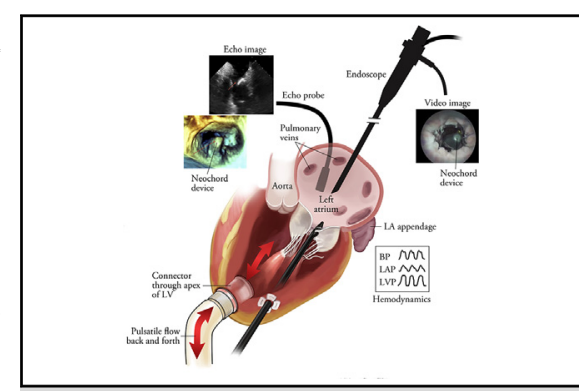

A dynamic cardiac biosimulator to train surgeons in beating-heart mitral valve repair.

\section{Central Message}

A dynamic animal heart biosimulator is evaluated during extruded polytetrafluoroethylene artificial chordae implantation into flail mitral leaflets under echocardiographic navigation Concomitant hemodynamic changes are displayed simultaneously.

\section{Perspective}

Simulation in cardiac surgery has become important for training with new technologic devices. Many simulators are low fidelity and may not represent true anatomy or physiologic function. Herein, we describe a dynamic animal heart biosimulator that recapitulates normal valve and hemodynamic function. We tested the utility by implanting extruded polytetrafluoroethylene chordae into redundant mitral leaflets under echocardiographic navigation followed by simultaneous videoscopy.

See Editorial Commentary page 156
In the past, cardiac surgeons and cardiologists learned the technical aspects of new devices first in the operating room and/or catheterization laboratory. With the rapid evolution of transcatheter aortic valve replacement and aortic

\footnotetext{
From the ${ }^{a}$ LifeTec Group, Eindhoven, The Netherlands; ${ }^{b}$ Department of Cardiac Surgery, Medicover Hospital, Warsaw, Poland; ${ }^{\mathrm{c} C l i n i c}$ of Cardiac and Vascular Diseases, Faculty of Medicine, Vilnius University, Vilnius, Lithuania; and ${ }^{\mathrm{d}}$ Department of Cardiovascular Sciences, East Carolina Heart Institute, East Carolina University, Greenville, NC.

Received for publication June 8, 2017; revisions received Aug 13, 2017; accepted for publication Sept 5, 2017; available ahead of print Oct 23, 2017.

Address for reprints: W. Randolph Chitwood, Jr, MD, 146 East Longmeadow Rd, Greenville, NC 27858 (E-mail: chitwoodw@ecu.edu).

$0022-5223 / \$ 36.00$

Copyright (c) 2017 by The American Association for Thoracic Surgery

https://doi.org/10.1016/j.jtcvs.2017.09.011
}

vascular stent grafts, it became obvious that new technologic iterations would require physiologic and anatomic simulation training platforms that better represented clinical practice. To develop and apply beating heart transcatheter valve repair and replacement devices, companies generally have taught clinicians to gain expertise by using fairly low-fidelity simulation platforms before the first

$\square$ Scanning this QR code will take
you to a supplemental video for
the article.




$$
\begin{aligned}
& \text { Abbreviations and Acronyms } \\
& \begin{aligned}
\text { 2D } & =2 \text {-dimensional } \\
\text { 3D } & =3 \text {-dimensional } \\
\text { ePTFE } & =\text { extruded polytetrafluoroethylene }
\end{aligned}
\end{aligned}
$$

clinical applications. These systems provided the user with limited, unrealistic tactile feedback along with minimal visual control. For this reason, new generations of simulators that use biological organs have emerged that recreate realistic anatomic and hemodynamic cardiac conditions. $^{1-7}$

Herein, we describe a dynamic heart simulator (LifeTec Group, Inc, Eindhoven, The Netherlands) that presents an effective method for learning and perfecting intracardiac valve procedures that require Cartesian $\mathrm{X}-\mathrm{Y}-\mathrm{Z}$ plane navigation with proficiency in hand/eye coordination. This dynamic ex vivo beating heart biosimulator combines the benefits of real-time echocardiographic imaging, videoscopic vision, and hemodynamic changes during off-pump mitral valve repairs via the use of extruded polytetrafluoroethylene (ePTFE) artificial chord implantations.

\section{TECHNIQUE}

Although the biosimulator can be used to evaluate any type of intracardiac device, procedure, and/or other types of structural heart disease, our goal was to train surgeons to perform off-pump transventricular mitral valve repairs on prolapsed or flail mitral valve leaflets. The ex vivo heart simulator was an optimal tool for preclinical training because of the combined benefits of bimodal intracardiac visualization, pathologic and hemodynamic replication, operative rehearsal, and postprocedure skill assessment. The DS1000 system (NeoChord, Inc, St Louis Park, Minn) is a commercially available device that is intended to treat patients having mitral valve regurgitation resulting from prolapsed or flail mitral valve leaflets that are secondary to ruptured or elongated native chordae tendineae. This technology was used as the control technology to assess the preclinical teaching potential using this biosimulator. ${ }^{8}$

\section{The Dynamic Cardiac Biosimulator}

The biosimulator is composed of a pulsatile dynamic fluid system that is attached to an explanted porcine heart (Figures 1 and 2). The left ventricle is pressurized cyclically to clinical levels by a pulse duplicator system, attached through a sealed apical cannula. Saline is used as a working fluid to allow intracardiac videoscopic visualization. Figure 3 shows the DS1000 system being inserted into the left ventricle near the apex. The transesophageal echo probe is placed on the posterior left atrium. Figure 4 shows the surgeon and anesthesiologist working together to navigate the device to deploy ePTFE chordae into the flail mitral leaflet.
Cardiac chamber pressures are monitored constantly and can be regulated by adjusting pre- and afterload modules to the desired in vivo clinical levels. As an example, in the presence of a flail mitral valve leaflet, left atrial pressures increase while systemic blood pressure falls. By restoring normal leaflet coaptation, repairing the leak, these pressures revert to normal levels (Figure 5).

\section{Imaging}

With the simulator, the working mitral valve is visualized simultaneously with a transesophageal echocardiographic probe placed directly on the left atrial posterior wall (Figures 2 and 3) and an intracardiac 2-dimensional (2D) endoscope that is passed into the left atrium (Figure 6). Throughout the procedure, a skilled echocardiographer manipulates the echo probe to render ideal 2D, multiplane images, displaying 2 orthogonal 2D planes (long axis and mitral commissure) simultaneously (Figure 7). In addition, 3-dimensional (3D) surgeon's en face views are displayed. All main procedure steps can be performed under echo guidance alone and include (1) device insertion and passage through left ventricular cavity, subvalvular apparatus, and mitral annular plane (2D multi-plane mode); (2) positioning the device in reference to the prolapsing segment (3D zoom mode with surgeon's en face view); (3) leaflet capture, device application, artificial chord attachment, and evaluation of the anatomical (3D zoom mode with en face view) and functional aspects of the repair (2D color Doppler mode only possible using heparinized blood in the simulator) (Figures 7 and 8). At first, the surgeon performs the procedure solely under echo guidance, as this most closely recapitulates the actual clinical scenario. Thereafter, by comparing simultaneous images between echo and intracardiac videoscopy, the quality of beating heart mitral valve repair can be assessed.

\section{Dynamic Mitral Repair: ePTFE Artificial Chordae Implantation}

Before the ex vivo porcine heart is attached to the simulator, native chordae tendineae to the middle scallop $\left(\mathrm{P}_{2}\right)$ of the posterior leaflet are cut in the porcine heart, rendering a flail leaflet (Figure 6). After the cardiac pressurization and pulsatile flow has been achieved, echo and direct intracardiac video images are displayed simultaneously, demonstrating the regurgitant dysfunction.

Once the pathology has been confirmed, a series of deep pledgeted purse string sutures are placed posterior and lateral to the cardiac apex along an orthogonal trajectory axis with the mitral annular plane. Under 2D echocardiographic multiplane guidance, the DS1000 system shaft is passed into the ventricle through the purse string (Figure 3). Thereafter, the device is navigated through the ventricular cavity, past the subvalvular apparatus, and toward the mitral valve. After the instrument tip has traversed 


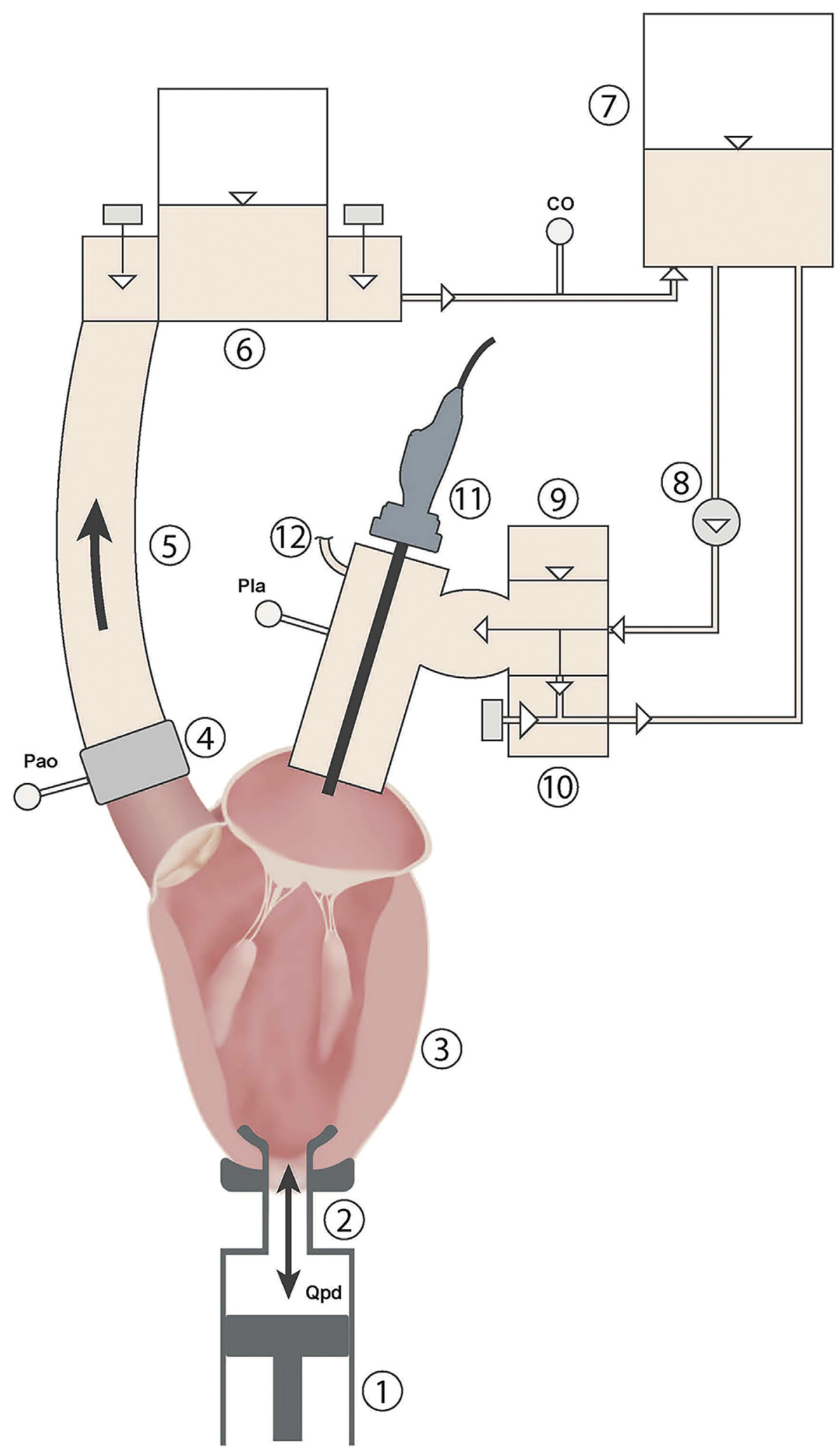

FIGURE 1. Schematic diagram of the dynamic cardiac biosimulator. (1) Pulse duplicator with a computer-controlled piston pump capable of replicating physiologic flow and ventricular pressure. (2) Apical cannula connecting the pulse duplicator to the left ventricle chamber. (3) Porcine heart (4) Aortic cannula connected to the ascending aorta. (5) Compliant silicone tube that mimics aortic compliance. (6) Afterload module comprised of two adjustable resistances and a compliant air tank. (7) Service reservoir. (8) Centrifugal pump used to provide fluid to the preload module. (9) Preload module composed of a small air tank and a silicone tube to mimic venous compliance. (10) Adjustable Starling resistor to ensure that atrial pressures are maintained at physiologic levels. (11) Endoscope with high definition camera to video the mitral valve. (12) Atrial cannula to convey the preload fluid to the left atrium. 


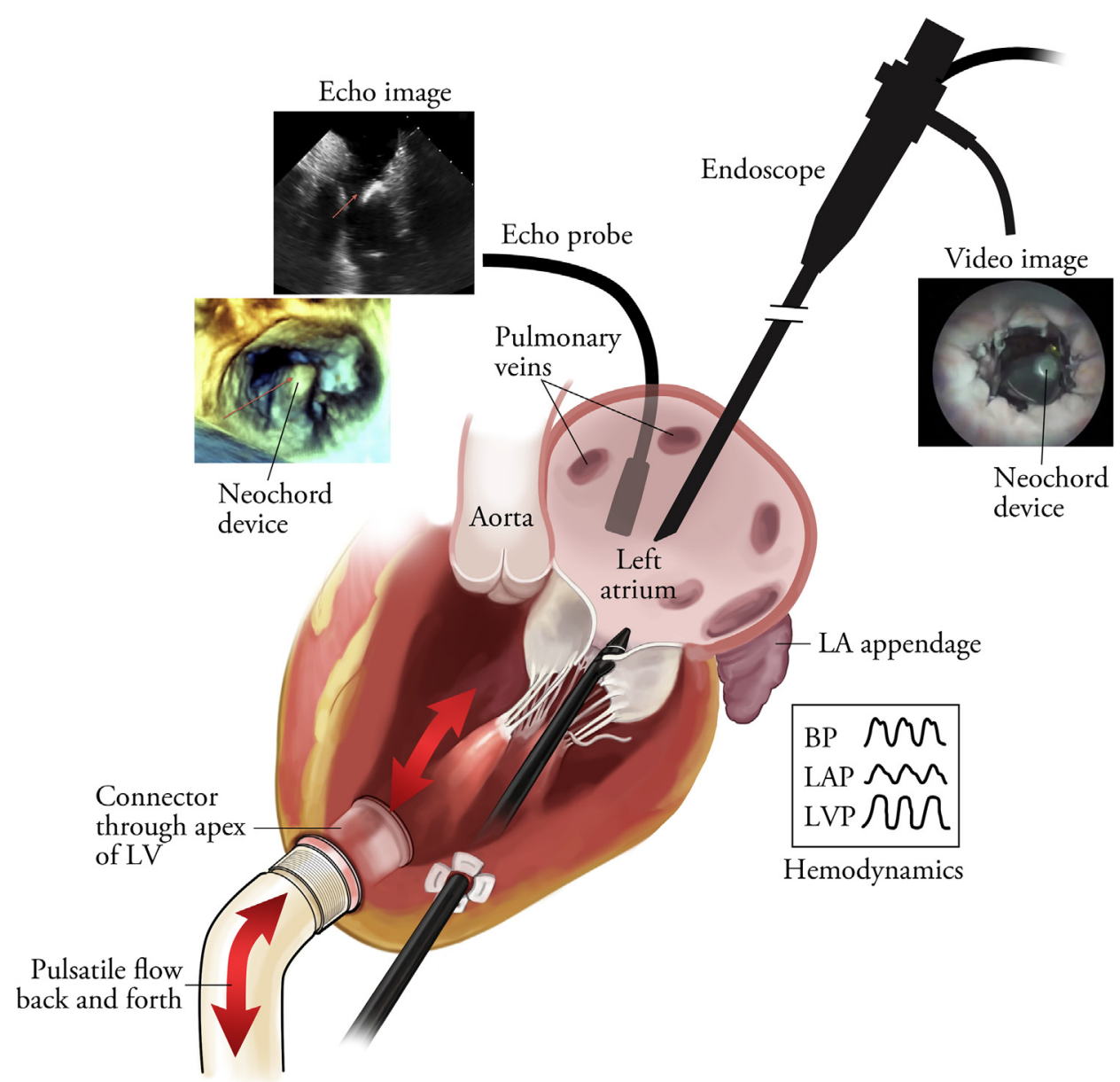

FIGURE 2. Graphic representation of the simulator during the artificial chordae placement procedure. The echocardiographic probe placed on the posterior left atrial wall. Hemodynamic parameters are measured simultaneously with endoscopic and echo visualization. $L A$, Left atrium; $L V$, left ventricle; $B P$, blood pressure; $L A P$, left atrial pressure; $L V P$, left ventricular pressure.

the mitral annulus, echo-guided, hand-driven X-Y-Z coordinate manipulation aligns it with the flail leaflet segment. At this point, the device jaws are opened so that leaflet edge resides within the device tip (Figures 6 and 8). In addition to echocardiographic confirmation, the DS1000 system includes a display monitor, which confirms adequate engagement. It includes fiber optic lights that transilluminate the leaflet tissue. When the flail or prolapsed mitral leaflet is engaged correctly, 4 "lighted windows" on the display monitor become illuminated simultaneously. This indicates that the DS1000 system tip has been oriented correctly and is closed over sufficient leaflet tissue to safely place an artificial chorda. If the closed tip is "skewed" along the leaflet surface, one or more indicator lights may not be lit, indicating less than ideal leaflet tissue engagement. Thus, simultaneous videoscopy, echocardiography, and fiber optic light confirmation are used to assess correct chorda placement along the leaflet edge.

After confirmation of mitral leaflet capture, a needle is advanced through the DS1000 system to penetrate the mitral leaflet 3 to $4 \mathrm{~mm}$ from the free edge. As the needle is withdrawn, through the device, a loop of preloaded ePTFE suture is captured and is passed through the mitral leaflet. Once the needle and loop have been exteriorized, the device tip is opened, releasing the mitral leaflet, and the entire device is withdrawn, exposing the "tails" of the artificial chord. Then, both suture tails are passed through the loop creating a "hitch knot," which is advanced to lock along the leaflet free edge.

At this point, the DS1000 system is reloaded with a new suture cartridge, and the procedure is repeated to place at least 3 artificial chordae along the prolapsing leaflet segment. Once all chords have been placed, lengths are adjusted to obtain good anterior-posterior leaflet coaptation and to assess the level of residual regurgitation using 2D color echo Doppler. Thereafter, exteriorized chords are anchored through a large pericardial pledget. Video 1 shows the procedure under both intracardiac and echocardiographic visualization.

\section{Learning, Quality, and Safety Metrics}

Although the biosimulator was used to train most surgeons using this device in Europe, definitive metrics were not collected. ${ }^{8}$ Nevertheless, it was clear to the trainers 

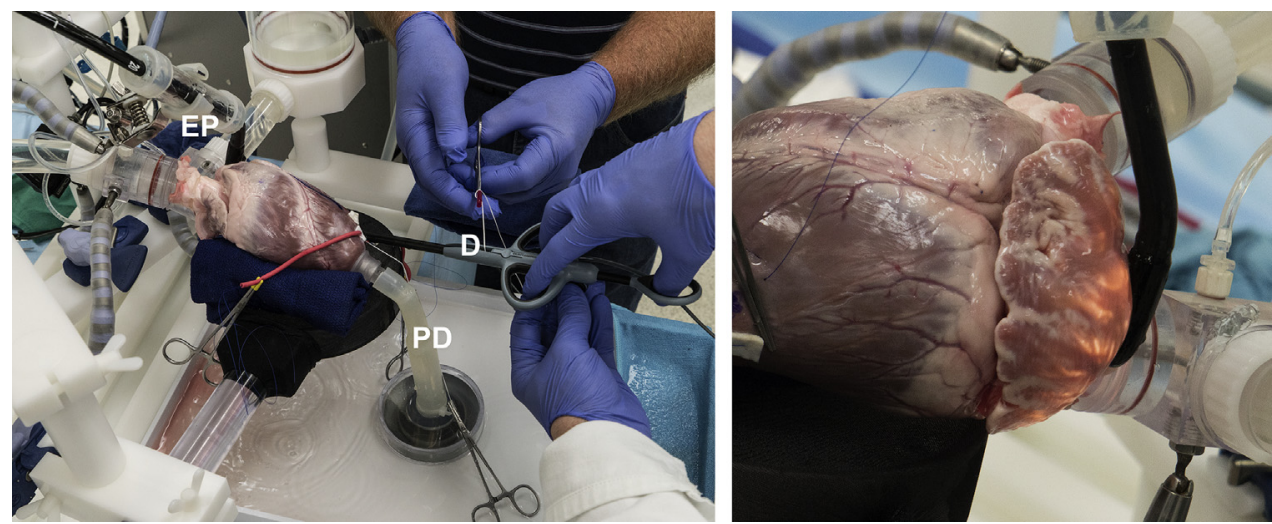

FIGURE 3. Left, The dynamic cardiac biosimulator system. Echo probe $(E P)$, pulse duplicator $(P D)$, and Neochord device $(D)$. Right, Transesophageal echo probe placed on the epicardium of the left atrial wall.

that with each attempt, surgeons' experienced iterative improvements in cardiac access, echocardiographic navigation, and accuracy of leaflet capture, PTFE chord deployment, and prolapse reduction. Lastly, the echocardiographic quality of the valve repair was assessed. Tables 1 and 2 list the learning, quality, and safety metrics that are being collected for the current US ReChord Trial.

\section{COMMENT}

Advanced training simulators should provide benefits for patients, surgeons, and device manufacturers by accelerating and flattening learning curves in a safe, reproducible, yet realistic environment. Biosimulation is likely to reduce safety and regulatory risks for companies who are introducing new devices into the market. Moreover, improved training methods such as full procedure simulations can enable skill benchmarking for specific devices and surgical procedures.

Hand-driven, low-fidelity, transcatheter valve simulators have been successful in training clinicians during the preclinical phase of their learning. Mitral valve models, which have been 3D printed from echocardiographic data, now are being used to train surgeons in minimally invasive repair techniques. ${ }^{9}$ Moreover, surgical robotic simulation has become paramount and is required for mastering the tele-manipulation based surgeon's console, which drives the wristed instruments. Valdis and colleagues ${ }^{10}$ demonstrated in a randomized controlled trial that operator skills improve markedly through robotic simulation. Robotic simulators, like the Mimic da Vinci Si Skills Simulator (MIMIC Technologies, Inc, Seattle, Wash), are able to define skill progression through "report card" metrics for accuracy, speed, and ergonomic motion.

Cardiopulmonary simulators are now used in resident training and provide realistic animal hearts for vascular cannulation and off-pump coronary surgery. ${ }^{711-14}$ Ex vivo simulation not only provides a natural operative environment but, at the same time, can emulate disastrous hemodynamic clinical conditions. A recent study showed that among surveyed cardiac training programs in the United States, simulation was extremely helpful in teaching cardiac surgical techniques. ${ }^{12}$ Currently, cardiothoracic residency programs have implemented the
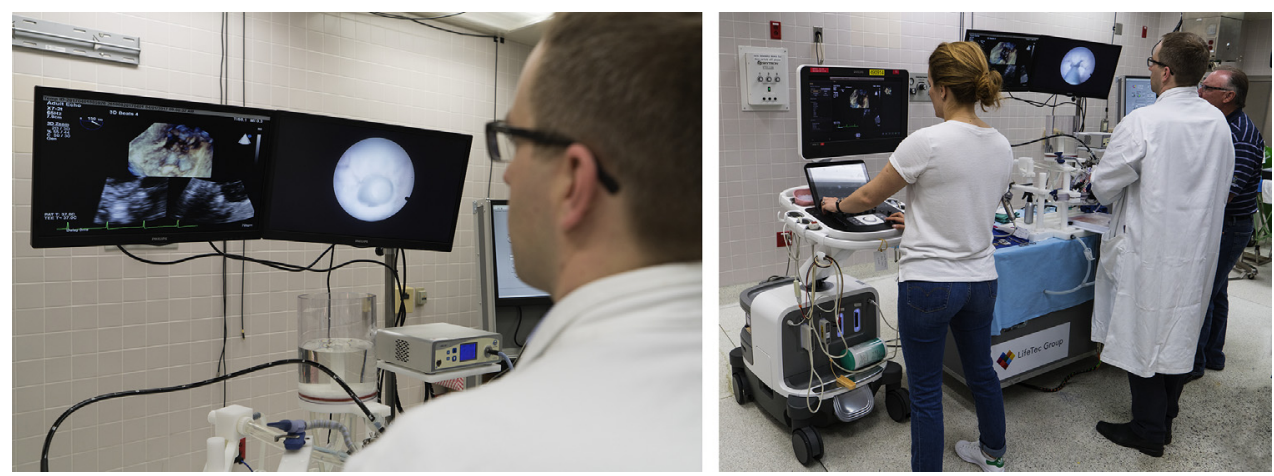

FIGURE 4. Left, The surgeon is navigating the device to engage the flail mitral valve leaflet with simultaneous 2-dimensional and 3-dimensional echo imaging well as intra-atrial videoscopy. Right, Echo probe manipulation and image evaluations are done simultaneously by an experienced cardiac anesthesiologist. After flail leaflet capture by the Neochord device, a extruded polytetrafluoroethylene chord is placed to reduce the prolapse under hemodynamic, videoscopic, and echocardiographic monitoring. 


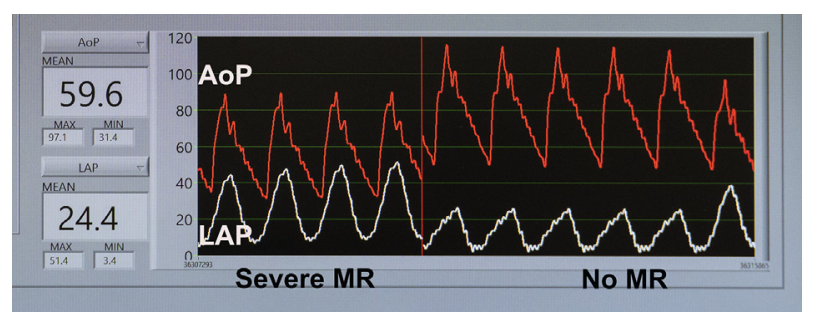

FIGURE 5. Hemodynamic tracings showing the effect of artificial chordae implantation into a flail mitral leaflet before and after correction of MR. Left, LAP and AoP with a prolapsing mitral valve and severe MR. Right, The same parameters after the mitral valve flail leaflet has been repaired with the artificial chordae. Note the decrease in LAP with concomitant increase in AoP after correction of the severe MR. AoP, Aortic systemic pressure; $L A P$, left atrial pressure; $M R$, mitral regurgitation.

requirement of 20 hours of simulation training for certification by the American Board of Thoracic Surgery. ${ }^{15}$ Thus, the question of "Simulation in cardiothoracic training: Where do we stand?" that Trehan posed in $2014^{16}$ is being answered.

The present high-fidelity biosimulator will allow clinicians to perfect skills to perform transcatheter and/or surgical valve procedures in a more natural milieu. Leopaldi and colleagues ${ }^{1}$ developed an earlier prototype of the present biosimulator, which employed animal hearts and replicated physiologic hemodynamics. The current system allows for continual echocardiographic and videoscopic cardiac valve visualization and also is compatible with fluoroscopy. ${ }^{2-4}$ In addition, this platform can be connected to patient-specific vascular models to replicate many transcatheter procedures. The hemodynamic performance of this simulator has been validated to provide near normal mitral valve function that is representative of in vivo conditions. ${ }^{4}$ In addition, physiologic leaflet coaptation was demonstrated without mitral insufficiency, unless there was previous chordal disruption. Although this biosimulator was used to recapitulate mitral valve dysfunction requiring valve repair, the platform can model other heart valve structural changes and can be used with other devices. Also, several types of fluids can be circulated in this platform, including heparinized blood. In our report, we employed a saline solution, which allowed for concurrent visualization of $2 \mathrm{D} / 3 \mathrm{D}$ echocardiography with intracardiac videoscopy. As there is no mitral annular dilatation associated with the heart model, we could assess technique associated with prolapsed and flail leaflet repairs, independent of other factors that can cause mitral insufficiency. Thus, this biosimulator has been ideal for reproducing clinical situations that meet the intended use for which the NeoChord DS1000 system and other chordal implantation devices have been designed. ${ }^{17}$

The placement of ePTFE artificial chordae has been shown to be very effective in a large series of mitral valve repairs and with excellent long-term results. ${ }^{18}$ However, in these series chordal replacement was combined with an annuloplasty prosthesis in most circumstances. Recent American College of Cardiology/American Heart Association guidelines suggest that repair surgery is appropriate for asymptomatic patients with severe mitral insufficiency. ${ }^{19,20}$ Generally, these patients are being referred earlier, and we can assume that in many of the patients, disease progression has not yet resulted in a significantly dilated annulus. Therefore, it is reasonable to assume that by correcting the dysfunction associated with leaflet prolapse or flail, subsequent annular dilatation may be limited enough to render a stable repair. The early European experience with the DS1000 system was presented at the Society of Thoracic Surgeons Annual Meeting (2017) and showed safety, efficacy, and excellent repairs in patients out to 2 years, especially in posterior leaflet repairs. ${ }^{21}$ Currently, the Food and Drug Administration has approved the US pivotal trial (ReChord; ClinicalTrials.gov identifier: NCT02803957) as a randomized controlled design. Enrollment has been
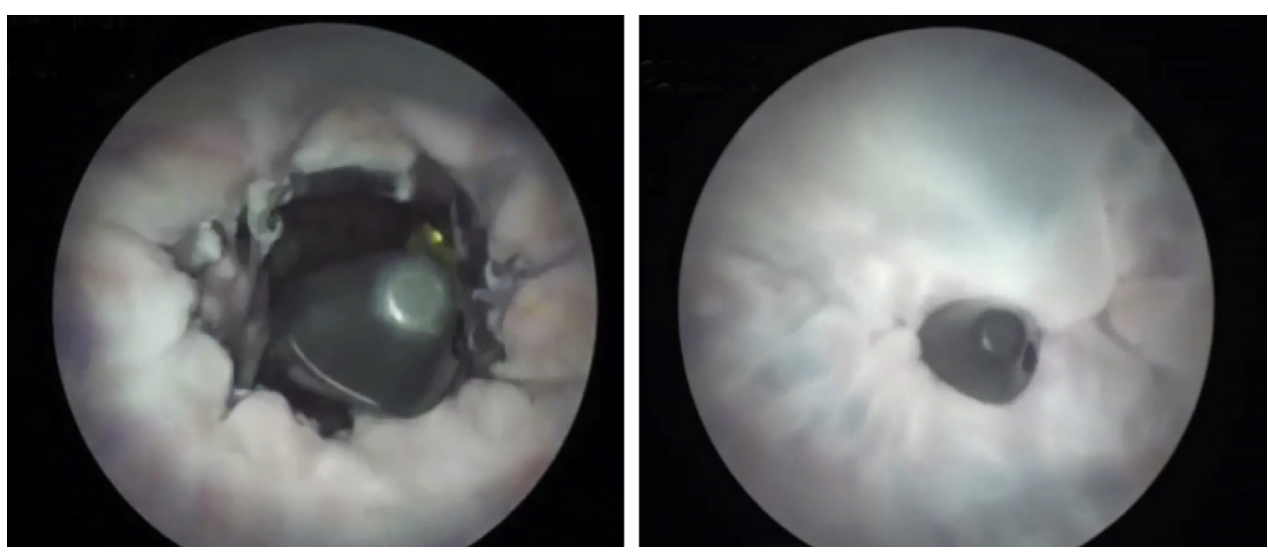

FIGURE 6. Left atrial endoscopic images. Left, prolapsing mitral valve with the DS1000 system positioned to capture the prolapsing leaflet segment. Right, The leaflet is captured and an artificial chorda is being deployed. 

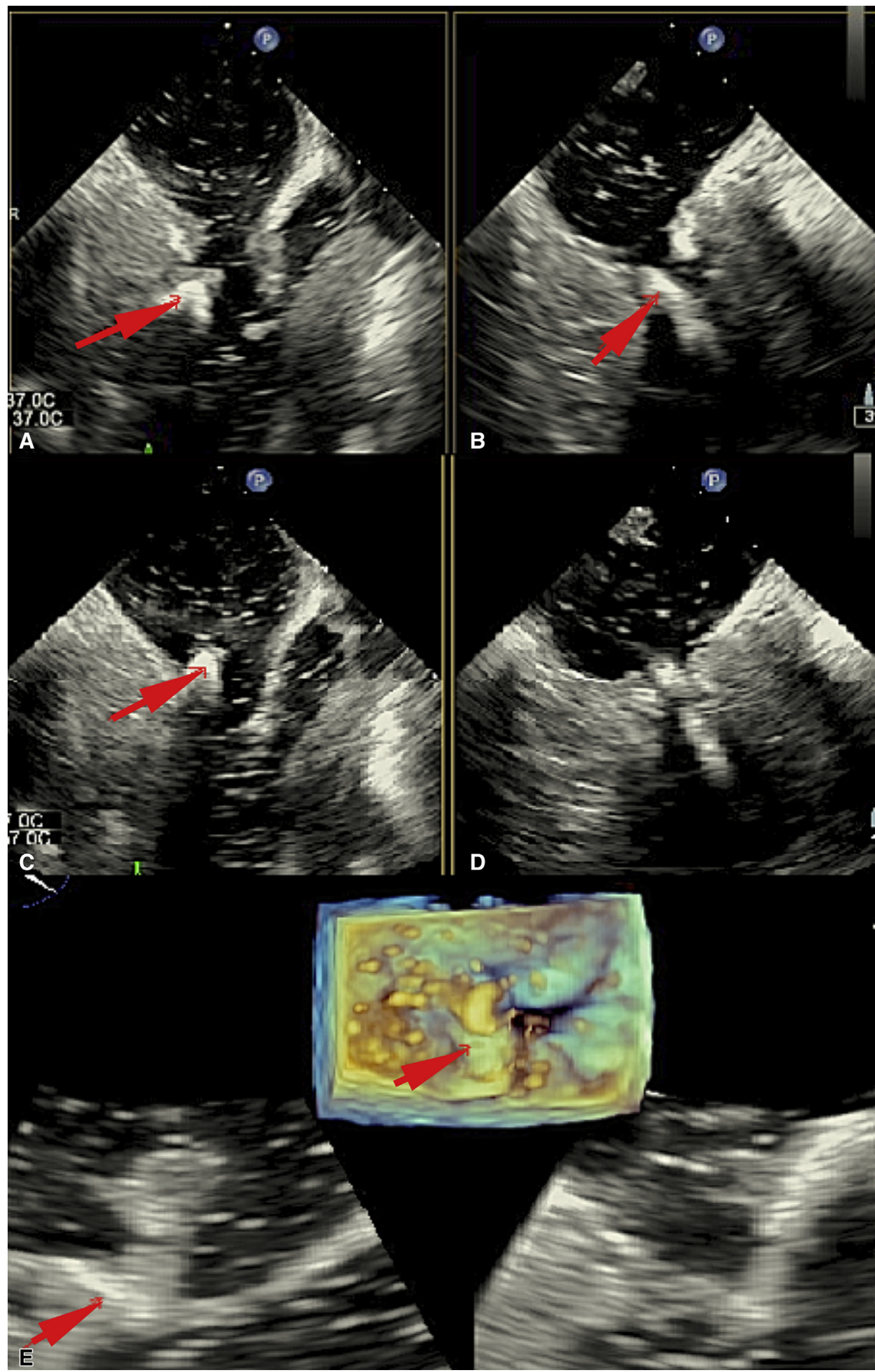

FIGURE 7. Representative 2-dimensional echo images during the procedure. X-plane modality with long axis and bi-commisural view. A and B, Device passage through left ventricular cavity and mitral valve plane. C and D, Arrow: Neochord device tip. E, Grasping of the leaflet with the open device (arrow) displayed simultaneously in 3-dimensional and 2-dimensional images. 


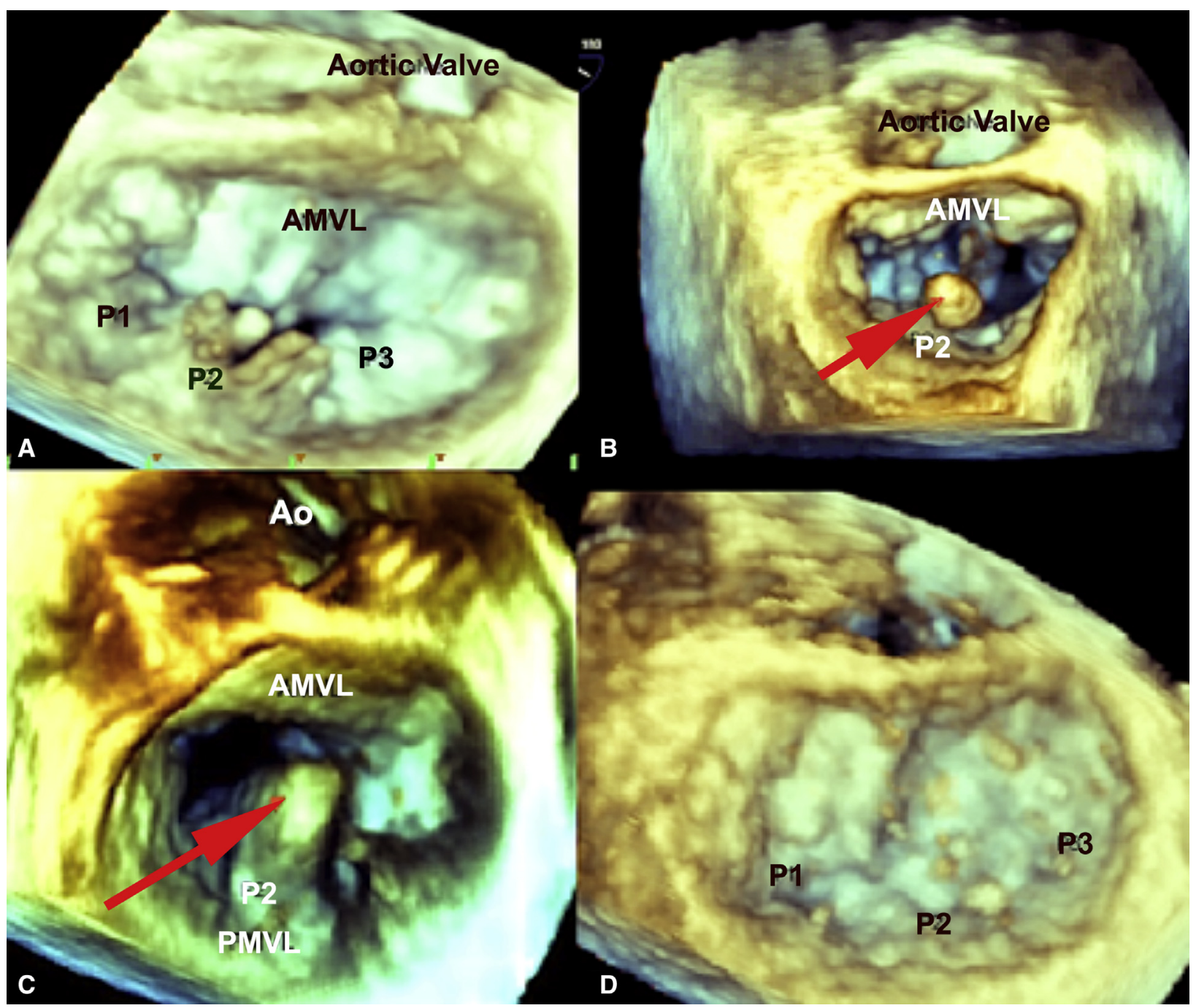

FIGURE 8. Three-dimensional zoom mode with en face view of the mitral valve: $\mathrm{A}, \mathrm{P}_{2}$ prolapse with multiple ruptured chords before the procedure; B, Neochord device in the left atrium - positioning adjacent to the prolapsing segment; C, P2 leaflet grasping; D, Final anatomical result after ePTFE chord implantation and length adjustment. $A M V L$, Anterior mitral valve leaflet; $P 1$, anteriormedial scallop; $P 2$, middle scallop; $P 3$, posteriolateral scallop; $A o$, aortic valve; $P M V L$, posterior mitral valve leaflet.

initiated in the ReChord trial, and the biosimulator platform is being used to train all trial investigators before their first operative cases. ${ }^{22}$ As mentioned previously, learning, quality, and safety metrics currently are being collected from surgeons training for the US ReChord clinical trial. In conclusion, simulation platforms have been developed to replicate and train to various types of hemodynamic

TABLE 1. Learning metrics for biosimulator training*

- Number of attempts and time to cross the mitral annular plane

- Number of attempts and time to capture the leaflet for the first time

- Leaflet capture quality (by number of indicator lights activated)

- Number of attempts and time to place the first chord

- Number of attempts and time to place multiple chords

- Time to anchor chords on the left ventricle

*At least 3 separate animal hearts are used for training each surgeon. and technical clinical challenges. The custom dynamic biosimulator, described herein, provides natural intracardiac mitral valve function that can be altered to emulate pathologic mitral regurgitation. This platform is

TABLE 2. Quality and safety metrics for biosimulator training

- 2-dimensional and 3-dimensional echocardiographic repair quality*

- Hemodynamic repair quality

- Chord detachment

- Incorrect topographic chord implantation

- Chord tension adequacy

- Leaflet trauma or perforation

- Atrial perforation

- Ventricular trauma

*Length of anterior and posterior leaflet coaptation; regurgitation quantitation. $\dagger$ Left atrial and ventricular pressure; cardiac output. 


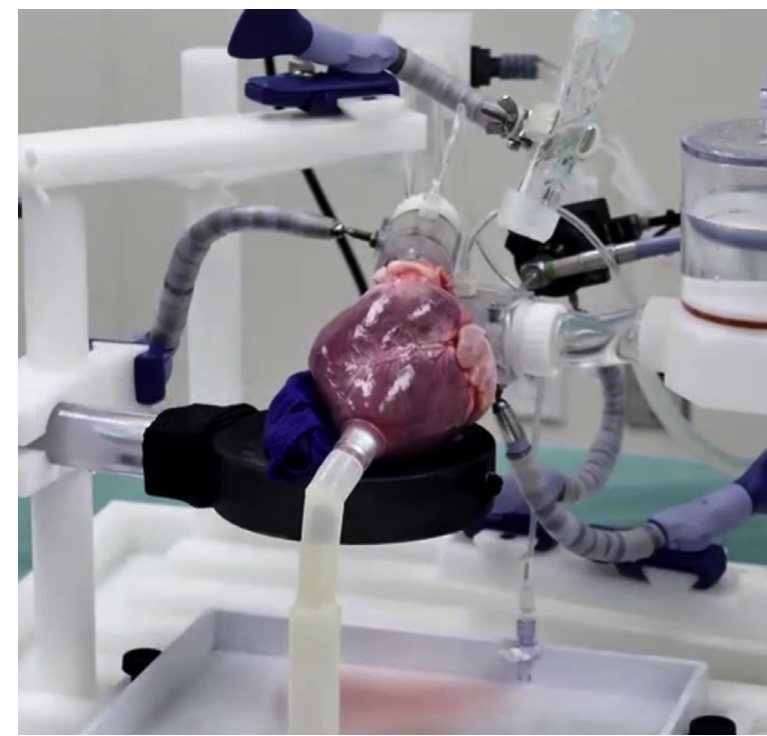

VIDEO 1. Dynamic biosimulatior: Off-pump mitral valve repair. Video available at: http://www.jtcvsonline.org/article/S0022-5223(17)31914-1/ fulltext.

ideal to train surgeons to place transventricular artificial chordae under echocardiographic visualization, for prolapsing or flail leaflets.

\section{Limitations}

The authors describe in detail this biosimulator and the use in training surgeons to replace ePTFE artificial chordae in prolapsing mitral valve leaflets under echocardiographic navigation. The paper is a descriptive one and is an attempt to bring this method of simulation to the awareness of surgeons who are training in transcatheter methods. The limitation of this paper is the lack of metrics from multiple learners using this training method. During the ReChord trial, each investigator will be evaluated with metrics that will assess improvements in navigational accuracy, procedure times, and ideal artificial chordae placement in a successive, iterative training method.

\section{Conflicts of Interest Statement}

Drs Chitwood, Jr, Drasutiene, Speziali, and Wrobel are consultants to Neochord Inc. Dr Leopaldi and S. van Tuijl are/or were employees of the LifeTec Inc. Neochord, Inc, funded the use of the biosimulator.

\section{References}

1. Leopaldi AM, Vismara R, Lemma M, Valerio L, Cervo M, Mangini A, et al. In vitro hemodynamics and valve imaging in passive beating hearts. $J$ Biomech. 2012;45:1133-9.

2. Leopaldi AM, Vismara R, Gelpi G, Romagnoni C, Fiore GB, Redaelli A, et al. Intracardiac visualization of transcatheter aortic valve and valve-in-valve implantation in an in vitro passive beating heart. JACC Cardiovasc Interv. 2013;6:92-3.
3. Leopaldi AM, Vismari R, van Tuijl S, Redaelli A, van de Vosse FN, Fiore GB, et al. A novel passive left heart platform for device testing and research. Med Eng Phys. 2015;37:361-6.

4. Vismara R, Leopaldi AM, Piola M, Asselta C, Lemma M, Antona C, et al. In vitro assessment of mitral valve function in cyclically pressurized porcine hearts. Med Eng Phys. 2016;16:346-53.

5. Gelpi G, Romagnoni C, Vismara R, Mangini A, Contino M, Gianfranco BF, et al. Intracardiac visualization of transcatheter mitral valve repair in an in vitro passive beating heart. Circulation. 2015;132:e131-2.

6. Bouma W, Jainandunsing JS, Khamooshian A, van der Harst P, Mariani MA, Natour E. A new beating-heart mitral and aortic valve assessment model with implications for valve intervention training. Interact Cardiovasc Thorac Surg. 2017;24:169-72.

7. Ramphal P, Coore DN, Craven MP, Forbes NF, Newman SM, Coye AA, et al. A high fidelity tissue-based cardiac surgical simulator. Eur J Cardiothorac Surg. 2005;27:910-6.

8. Colli A, Manzan E, Zucchetta F, Bizzotto E, Besola L, Bagozzi L, et al. Transapical off-pump mitral valve repair with Neochord implantation: early clinical results. Int J Cardiol. 2016;204:23-8.

9. Nia PS, Heuts S, Daemen J, Luyten P, Vanier J, Hoorntje J, et al. Preoperative planning with three-dimensional reconstruction of patient's anatomy, rapid prototyping and simulation for endoscopic mitral valve repair. Interact Cardiovasc Thorac Surg. 2017;24:63-8.

10. Valdis M, Chu MW, Schlachta C, Kiaii B. Evaluation of robotic cardiac surgery simulation training: a randomized controlled trial. J Thorac Cardiovasc Surg. 2016;151:1498-505.

11. Feins RH, Burkhart HM, Conte JV, Coore DN, Fann JI, Hicks GL, et al. Simulation-based training in cardiac surgery. Ann Thorac Surg. 2017;103: 312-21.

12. Mokadam NA, Fann JI, Hicks GL, Nesbitt JC, Burkhart HM, Conte JV, et al Experience with the cardiac surgery simulation curriculum: results of the resident and faculty survey. Ann Thorac Surg. 2017;103:322-8.

13. Hicks GL, Gangemi J, Angona RE, Rahmpal PS, Feins RH, Fann JI. Cardiopulmonary bypass simulation at the boot camp. J Thorac Cardiovasc Surg. 2011; 141:284-92.

14. Smelt JLC, Phillips S, Hamilton C, Fricker P, Spray D, Nowell JL, et al. Simulator teaching of cardiopulmonary bypass complications: a prospective, randomized study. J Surg Educ. 2016;73:1026-31.

15. American Board of Thoracic Surgery. Operative requirements. Available at: https://www.abts.org/root/home/certification/requirements/operative-requirements. aspx. Accessed June 5, 2017.

16. Trehan K, Kemp CD, Yang SC. Simulation in cardiothoracic surgical training: where do we stand? J Thorac Cardiovasc Surg. 2014;147:18-24.

17. Gammie JS, Wilson P, Bartus K, Gackowski A, Hung J, D’Ambra MN, et al. Transapical beating-heart mitral valve repair with an expanded polytetrafluoroethylene cordal implantation device: initial clinical experience. Circulation. 2016;134:189-97.

18. David TE, Armstrong S, Ivanov J. Chordal replacement with polytetrafluoroethylene sutures for mitralvalve repair: a 25-year experience. J Thorac Cardiovasc Surg. 2013;145:1563-9.

19. Nishimura RA, Otto CM, Bonow RO, Carabello BA, Erwin JP III, Guyton RA, et al. 2014 AHA/ACC Guidelines for the Management of Patients With Valvular Heart Disease. J Am Coll Cardiol. 2014;63:2438-88.

20. Nishimura RA, Otto CM, Bonow RO, Carabello BA, Erwin JP III, Guyton RA, et al. 2014 AHA/ACC Guidelines for the Management of Patients With Valvular Heart Disease. Circulation. 2014;129:2440-92.

21. Colli A, Manzan E, Aidietis A, Rucinskas K, Bizzotto E, Besola L, et al. Early European experience with echo-guided transapical off-pump mitral valve with NeoChord implantation. Presented at Society of Thoracic Surgeons Annual Meeting. Houston: Texas; 2017.

22. Randomized Trial of the Neochord DS1000 System Versus Open Surgical Repair (ReChord), 2016, NCT02803957. Available at: https://clinicaltrials.gov/ct2/ show/NCT02803957. Accessed June 5, 2017.

Key Words: simulation, biosimulator, mitral, valve, chord, replacement 